\title{
UCHII Regions and Newly Born O-type Stars
}

\author{
Peter S. Conti ${ }^{1}$, Jeonghee Rho ${ }^{2}$, James Furness ${ }^{3}$ and Paul A. \\ Crowther ${ }^{3}$ \\ ${ }^{1}$ JILA, University of Colorado, Boulder CO 80309-0440 \\ e-mail:pconti@jila.colorado.edu \\ ${ }^{2}$ Spitzer Science Center, CalTech, Pasadena CA 91125 \\ email: rho@ipac.caltech.edu \\ ${ }^{3}$ Department of Physics and Astronomy, University of Sheffield, S3 7RH,UK \\ email: J.Furness@sheffield.ac.uk, Paul.Crowther@sheffield.ac.uk
}

\begin{abstract}
We have obtained Spitzer IRAC and MIPS mid-IR images of a sample of 43 radio selected UCHII region sources to ascertain $(a)$ whether the newly born $\mathrm{O}$ stars within are found with other stars in their birthplaces and $(b)$ the nature of the surroundings. 37 of the sources appear to be in small clusters, and 33 are found in connection with other hot star formation activity. Thus, for the most part, $\mathrm{O}$ stars are not born in isolation. Here we give examples of the mid-IR images of the various types of UCHII regions.
\end{abstract}

Keywords. stars: formation - ISM: HII regions - infrared: ISM

\section{Introduction}

UCHII regions are dense, highly compact $(<0.1 \mathrm{pc})$ radio sources of line and continuum hydrogen emission surrounding newly born O-type stars. All newly born $\mathrm{O}$ stars go through this phase, which results from the ionization of the hydrogen in the dense natal cocoon remaining immediately after the birth processes. The inner cocoon is composed of ionized hydrogen, while further out neutral and molecular compounds, along with extensive dust, are present. This dust cocoon is roughly ten times the size of the UCHII region and its extensive extinction renders direct detection of the central exciting star(s) difficult at wavelengths shorter than the mid-IR (MIR).

Eventually, the cocoon material is dissipated by the central star's very high luminosity, its winds, and prodigious Lyman continuum radiation. Then the central object is revealed to our view. It might be appropriate here to note that the nearby and well known Orion Trapezium Cluster is being observed long after its UCHII region phase.

While our eventual objective is to determine the nature of the central exciting star(s) and relate them to the evolution and interaction with the natal material, here we wish to consider the following simpler questions:

Are the central exciting sources single or multiple?

Is the formation of O-type stars isolated in space or is there evidence of other nearby activity, or other hot luminous stars (O-type or early-B)?

\section{Target acquisition}

We have obtained Spitzer MIR images of 43 relatively isolated UCHII regions in our Galaxy, extracted from the catalogs of Wood \& Churchwell (1989), Kurtz, et al. (1994), 


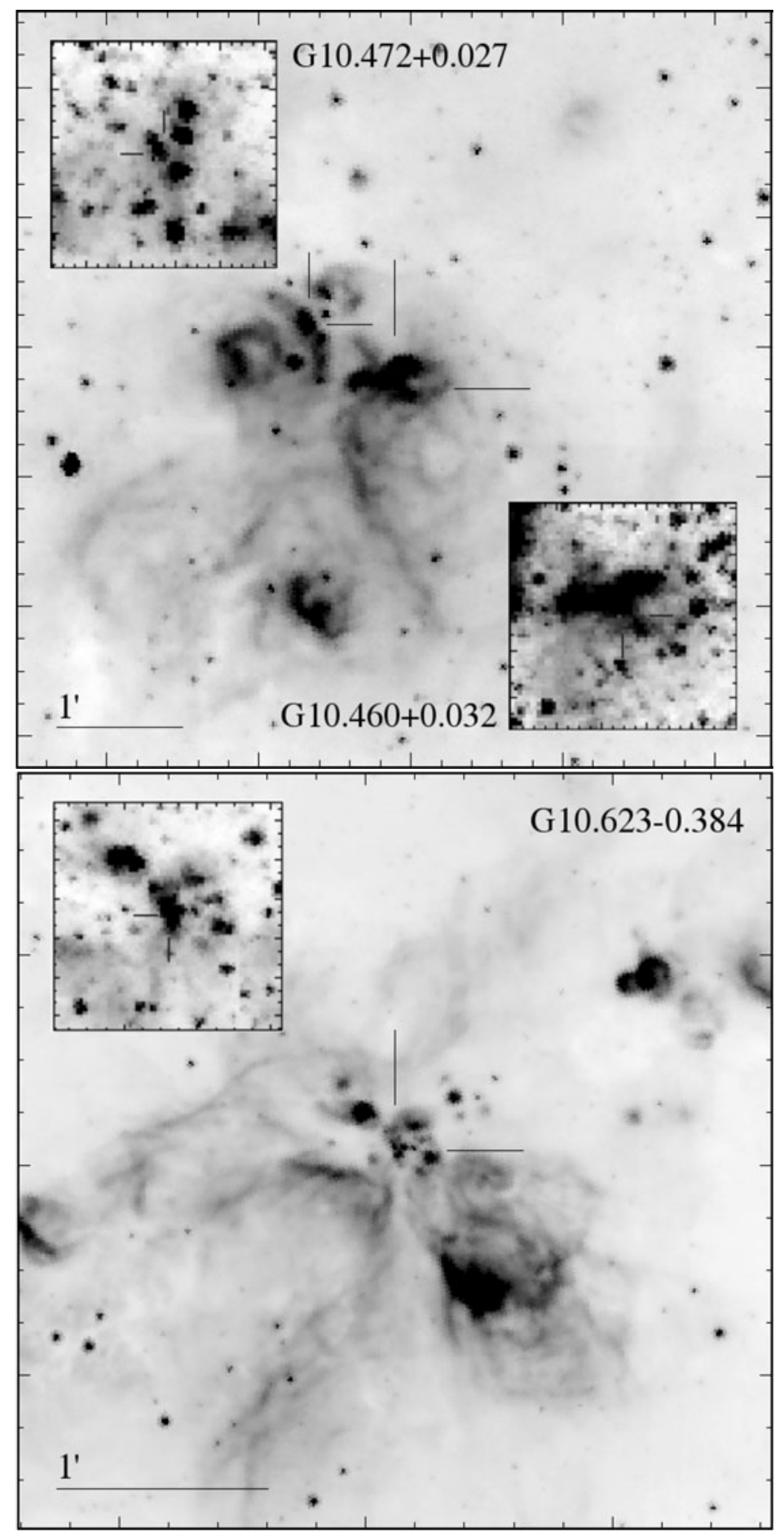

Figure 1. Spitzer/IRAC $8 \mu \mathrm{m}$ images of G10.460+0.032 and G10.472+0.027 (top) and G10.623-0.384 (bottom), with a scale of $10 \times 10$ pc for their adopted distance. Inset within these are $4.5 \mu \mathrm{m}$ images of the central $2 \times 2 \mathrm{pc}$ region presented at a higher spatial scale. 
and Crowther \& Conti (2003). The first two of these papers were radio surveys along the Galactic plane specifically searching for UCHII regions. Some 200 were found, nearly all of them not associated with giant HII regions which harbor many O-type stars. The latter paper presented MSX satellite MIR imaging data of 53 of these UCHII region sources. Here we consider 43 of the MSX list, utilizing the higher spatial resolution now available with the Spitzer instrument.

Generally speaking, UCHII regions are readily detected by radio surveys in uncrowded fields. They do exist in HII and GHII regions and clusters but they are difficult to identify there unless specific detailed searches have been carried out. For example, dozens are known in the GHII region W49A (de Pree et al. 1997). In our sample, only G49.4900.370 is found in a GHII region, namely within IRS2 of W51 (see also poster by Barbosa et al. in this volume).

The Spitzer IRAC data we present here is at wavelengths of $4.5 \mu \mathrm{m}$ and $8 \mu \mathrm{m}$, obtained at a spatial resolution of $\sim 2$ arcsec. We also have $24 \mu \mathrm{m}$ MIPS images of the same sources but they are not considered here given the limited discussion we can carry out in a short presentation such as this one. A more detailed and lengthy paper is being generated for publication which will consider all our data.

\section{Examples of Spitzer imaging}

We will present representative data for four UCHII regions, illustrating the wide divergence of MIR spatial morphologies. These figures are uniformly scaled to $10 \times 10 \mathrm{pc}$ at $8 \mu \mathrm{m}$ with an insert of $2 \times 2 \mathrm{pc}$ at $4.5 \mu \mathrm{m}$. The former can be used to identify star formation activity near to and associated with the UCHII region as indicated by dust emission. The shorter wavelength samples the stellar content and is used to investigate possible multiplicity of the exciting object.

The distances of the UCHII regions are derived from the radio kinematic method whereby the radial velocities are used to infer that number from a Galactic rotation model and an assumption of purely circular galacto-centric motion. Our distance determination is described in Furness et al. (2008) It needs to be kept in mind that while these values are, so far, the only ones available, there could be problems with the near side/far side ambiguity for objects within the Solar circle. Additionally, if random motions are present, the derived distances could well be in error. Extensive studies of GHII regions using their detected and classified O-type stars, and spectroscopic parallaxes to obtain distance estimates, have frequently led to numbers which are discrepant with the published radio kinematic distances (e.g., Damineli et al. 2005).

\subsection{G10.460+0.032 and G10.472+0.027}

In the upper panel of Figure 1, we show a close pair of UCHII regions, each one within a somewhat larger region of hot star activity as evidenced by the extensive dust emission. They have similar kinematic distances. These two active regions are separated by $2 \mathrm{pc}$ (in projection) and appear to be spatially connected. Another active region is found to the south at $5 \mathrm{pc}$, which may also be associated with the pair.

The $2 \times 2$ pc insert clearly shows the multiplicity of stellar images surrounding each UCHII region strongly suggesting that each resides in a small cluster of size about a parsec. We infer from these data that both UCHII regions are in clusters and other star birth activity is found nearby. 


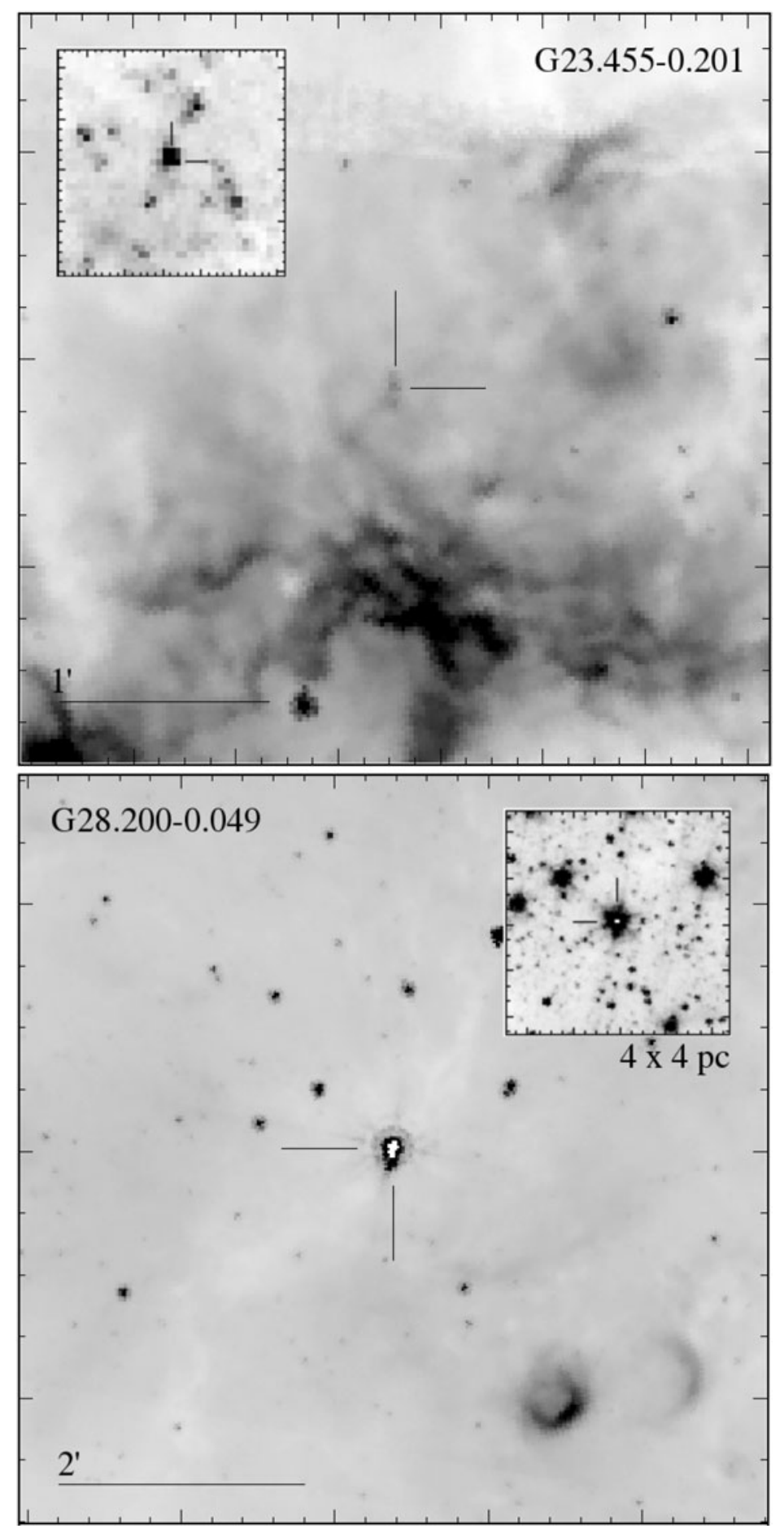

Figure 2. Spitzer/IRAC $8 \mu \mathrm{m}$ images of G23.455-0.201 (top) and G28.200-0.049 (bottom), with a scale of $10 \times 10 \mathrm{pc}$ for their adopted distance. Inset within these are $4.5 \mu \mathrm{m}$ images of the central $2 \times 2 \mathrm{pc}$ region $(4 \times 4 \mathrm{pc}$ for G28.200-0.049) presented at a higher spatial scale. 


\subsection{G10.623-0.384}

In the lower panel of Figure 1, we present an image of an UCHII region in the midst of a cluster of stars, along with a much stronger active region (G10.598-0.383) a few pc to the SW. While we do not have kinematic information concerning this object it appears to be enveloped in the same dusty material surrounding the UCHII region. We thus have evidence for a cluster and for nearby activity.

\subsection{G23.455-0.201}

As indicated by the upper panel of Figure 2, this UCHII region sits on the very outskirts of a very strong IR source, G23.437-0.209, which dominates the dust emission. Kim \& Koo (2001) provide low resolution 21-cm radio data suggesting this dust source is also a strong HII emitter. The UCHII region appears unresolved at $4.5 \mu \mathrm{m}$ and there is no evidence for a cluster surrounding this object. Without kinematic information for the strong dust source we cannot say if they are connected. This object might be considered to be isolated.

\subsection{G28.200-0.049}

The lower panel of Figure 2 shows very strong IR source with a fainter very red companion less than a parsec to the south, but there is no evidence for any cluster nearby. The UCHII region also appears isolated, one of the very few in our survey.

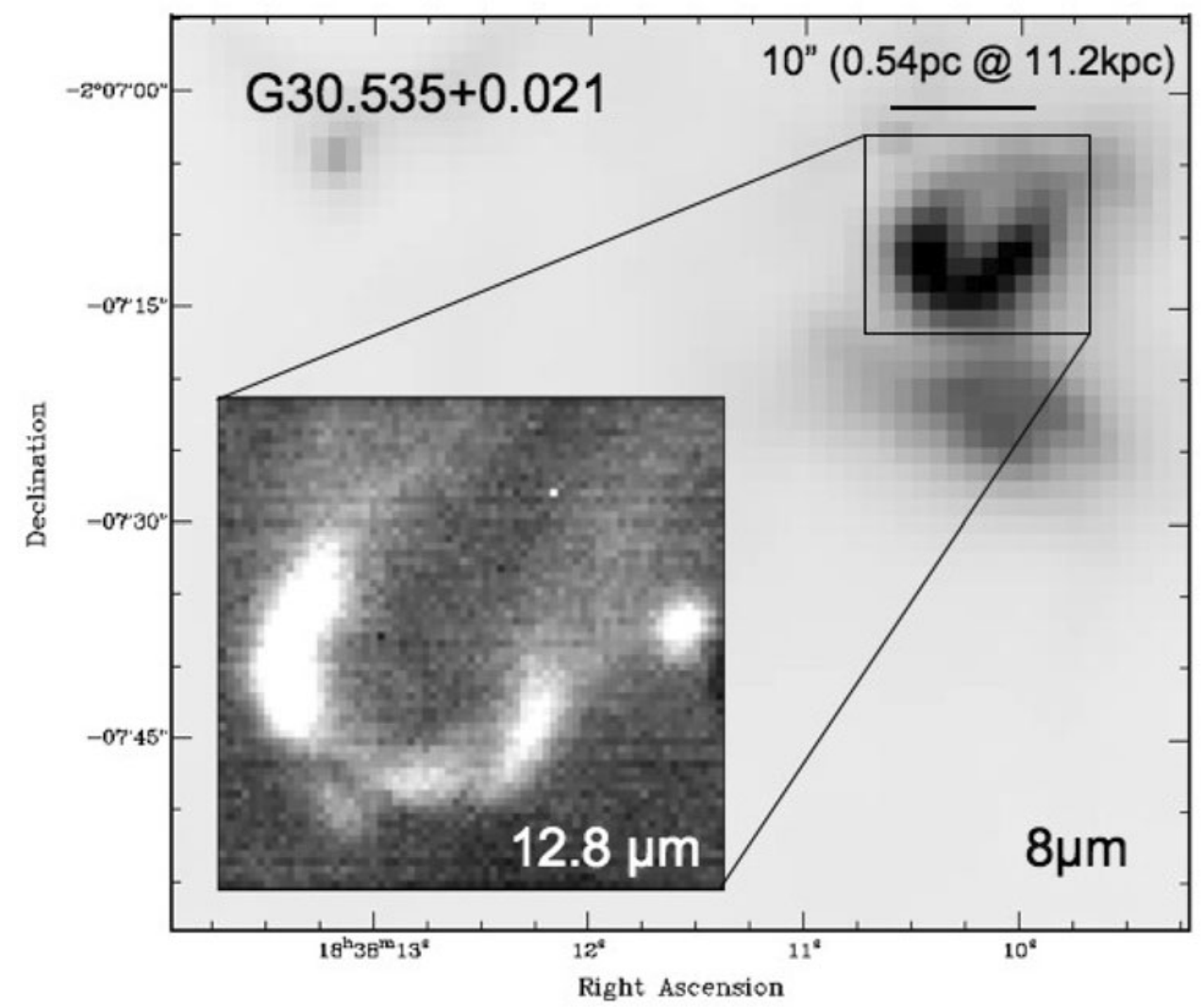

Figure 3. Spitzer/IRAC $8 \mu \mathrm{m}$ image of G30.535+0.021 $(3.6 \times 3.6 \mathrm{pc}$ at $11.2 \mathrm{kpc})$ together with a higher spatial resolution VLT/VISIR image at $12.8 \mu \mathrm{m}$ (inset). 


\begin{tabular}{crcl}
\hline Source & $\mathrm{d}(\mathrm{kpc})$ & Cluster? & Environment \\
\hline G10.460+0.032 & 5.8 & yes & similar nearby \\
G10.472+0.027 & 5.8 & yes & similar nearby \\
G10.623-0.384 & 17.5 & yes & others nearby \\
G23.455-0.201 & 9.0 & no & stronger nearby \\
G28.200-0.049 & 3.4 & no & isolated \\
\hline
\end{tabular}

Table 1. Summary of properties for UCHII regions discussed here

\section{Results}

In Table 1 we compare the properties of the UCHII regions considered here. Turning now to the entire sample of 43 sources, of which we have shown only four, we find that 38 of them $(86 \%)$ have evidence of being connected to a small cluster of stars. This conclusion comes from careful examination of the $4.5 \mu \mathrm{m}$ wavelength images similar to those shown in our figures here. Of the other 5 sources, a couple of them appear to be at large distances such that duplicity might remain undetected. Examination of the environments of the 43 sources, using the $8 \mu \mathrm{m}$ wavelengths, indicates that $32(72 \%)$ appear to be connected to other nearby star formation activity. Star formation activity thus appears not to be isolated in most cases.

The very small fraction of UCHII region O-type stars that appear to be truly single is roughly consistent with recent extensive star counts of isolated (4\%) field stars (e.g., Parker \& Goodwin (2007) and references therein). Also, from the examinations of the surroundings of UCHII regions, it would appear that most of them have nearby, although not extensive, star formation activity.

Of course, the $\sim 2$ arcsec spatial resolution of IRAC at $8 \mu \mathrm{m}$ corresponds to a physical scale of $0.1 \mathrm{pc}$ at a distance of $10 \mathrm{kpc}$, which is comparable to the size of UCHII regions, so one would need to observe these regions at much greater spatial resolution to directly probe within the ionized region. Fortunately, efficient MIR imagers are now available at current $8-10 \mathrm{~m}$ ground-based telescopes, such as MICHELLE at Gemini-North and VISIR at the ESO Very Large Telescope. One such example is presented in Fig. 3, showing a $12.8 \mu \mathrm{m}$ VLT/VISIR image of G30.535+0.021 obtained in July 2007 at a spatial resolution of $\sim 0.4$ arcsec, corresponding to $0.02 \mathrm{pc}$ at the $11.2 \mathrm{kpc}$ distance of G30.535+0.021, sampling the warm dust within the UCHII region. The dust spatial morphology is reminiscent of $2-6 \mathrm{~cm}$ radio continuum contours from Wood \& Churchwell (1989) obtained at a similar resolution.

PSC would like to thank JPL and the NSF for support in the investigations reported here. VLT/ISAAC observations were obtained at ESO Paranal Observatory under programme 079.C-0093(A).

\section{References}

Crowther, P. A. \& Conti, P. S. 2003, MNRAS, 343, 143

Damineli, A., Blum, R. D., Figueredo, E., \& Conti, P. S. 2005, in: R. Cesaroni, M. Felli, E. Churchwell, \& M. Walmsley, (eds.), Massive Star Birth: A Crossroads of Astrophysics (Cambridge: CUP), Proc IAU Symp. 227, 407

de Pree, C. G., Mehringer, D. M. \& Goss, W. M. 1997, ApJ, 482, 307

Furness, J., Crowther, P. A., Conti, P. S., Rho, J. \& Goodwin, S. P. 2008, MNRAS, submitted Kim, K.-T. \& Koo, B.-C. 2001, ApJ, 549, 979

Kurtz, S., Churchwell, E., \& Wood, D. O. S. 1994 ApJS, 91, 651

Parker, R. J. \& Goodwin, S. P. 2007, MNRAS, 380, 1271

Wood, J. J. \& Churchwell, E. 1989, ApJS, 69, 831 


\section{Discussion}

ZINNECKER: Your last conclusion that massive stars are not born in isolation is important, but probably premature in view of your limited sample. If we find one truly isolated massive star (not a runaway) it may be more significant for the understanding of massive star formation than discovering another 10 embedded clusters of massive stars. Larger unbiased surveys, perhaps in the LMC, are needed to settle this issue (cf. Zinnecker \& Yorke 2007 ARA $\& A 45$, 481, their section 2.3).

Conti: The sample of 43 objects is nearly a fifth of the known radio selected objects outside of GHII regions.

Crowther: In addition to MIR Spitzer data, we can also use the ratio of the FIR to radio emission to enable the stellar content of UCHII regions to be determined. In general, most sources are consistent with star clusters (Kroupa) of which some appear isolated. This does not necessarily mean they are isolated but perhaps associated only with a low mass star cluster (Furness et al. 2008; Parker \& Goodwin 2007).

OEY: To clarify: you said that 38 of the 43 UCHII regions show small clusters. Does that mean that 5 are isolated?

Conti: The number of non-isolated UCHII was 32 out of 43, thus the word "many". Those in detected clusters are 37 of 43 . Some resident clusters might have too small a diameter to be detected with the Spitzer instruments. These might show up in the improved ground based MIR data I presented at the very end.

OEY: Also, to follow Zinnecker's point, my student, Joel Lamb and I are indeed studying the field of $\mathrm{O}$ stars in the SMC to constrain the numbers of $\mathrm{O}$ stars found in isolation.

SKINNER: In the high resolution $12 \mu \mathrm{m}$ VLT images that you showed, can you speculate on what the faint emission extending away from the bright central object might be? Stellar or non-stellar?

Conti: I would imagine it is locally heated dust. The scale size shown was something like $0.01 \mathrm{pc}$, much more spatially detailed than the Spitzer data.

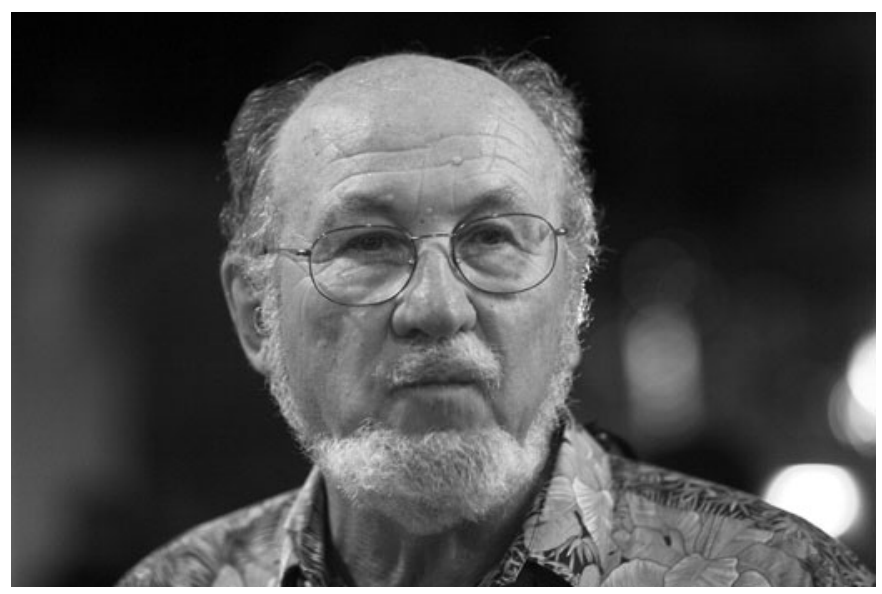

Peter Conti. 


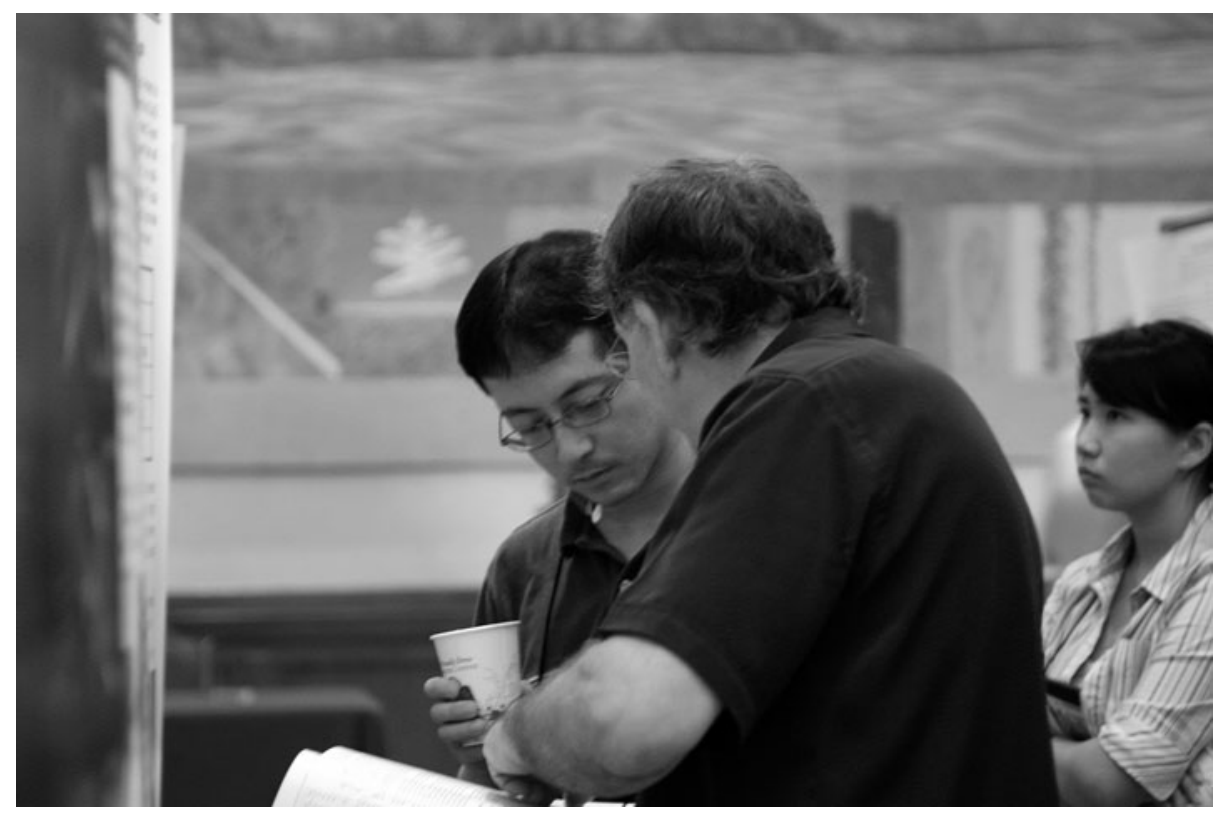

Takashi Hattori discusses with Mike Dopita.

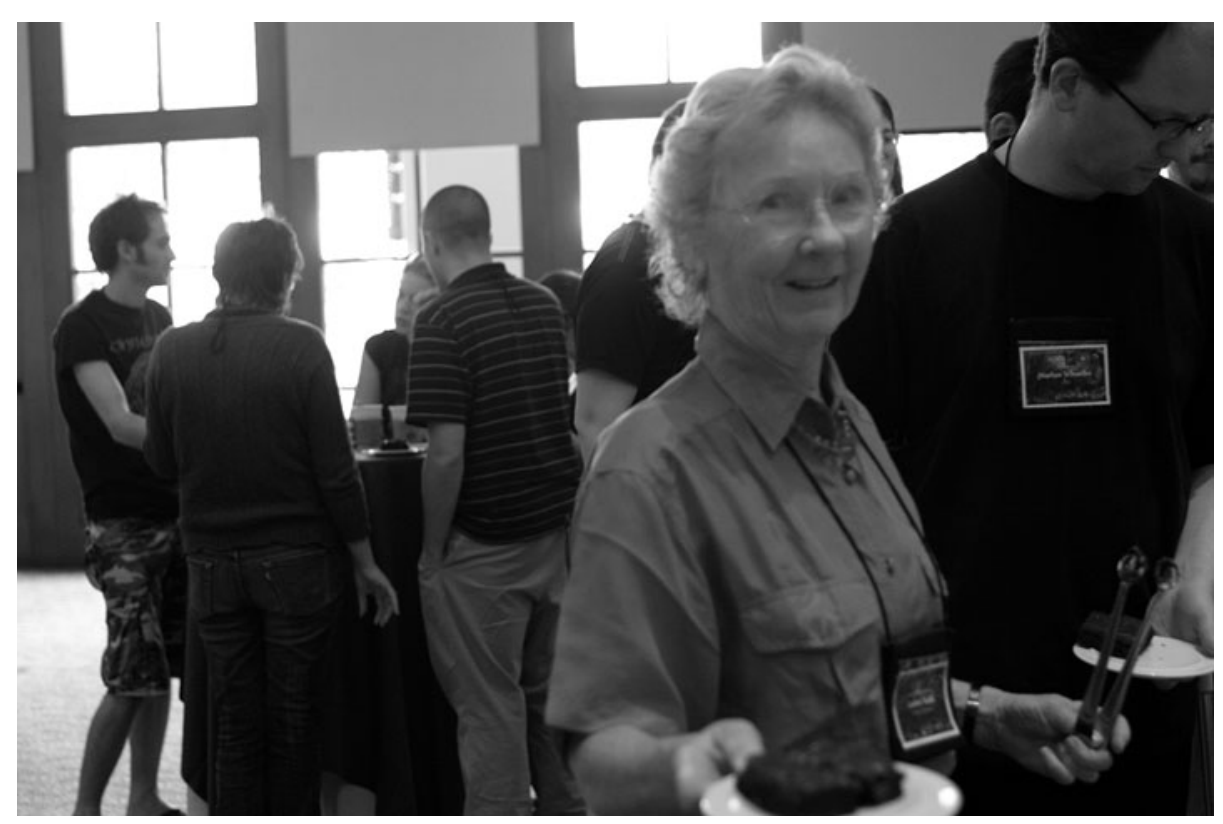

Lindsey Smith. 